\title{
EVOLUÇÃO E PERIFERIZAÇÃO DA COVID-19 NA ÁREA URBANA DE SANTA MARIA, RS: TRAÇANDO PADRÕES ESPACIAIS
}

\section{EVOLUTION AND PERIPHERALIZATION OF COVID-19 IN THE URBAN AREA OF SANTA MARIA, RS: SETTING SPATIAL PATTERNS}

\author{
Maurício Rizzatti \\ Doutorando em Geografia - Programa de Pós-Graduação em Geografia \\ Universidade Federal de Santa Maria \\ geo.mauricio.rizzatti@gmail.com \\ Pedro Leonardo Cezar Spode \\ Doutorando em Geografia - Programa de Pós-Graduação em Geografia \\ Universidade Federal de Santa Maria \\ pedrospode@gmail.com \\ Natália Lampert Batista \\ Pós-Doutoranda em Geografia - Programa de Pós-Graduação em Geografia \\ Universidade Federal de Santa Maria \\ natilbatista3@gmail.com \\ Douglas Bouvier Erthal \\ Mestrando em Geografia - Programa de Pós-Graduação em Geografia \\ Universidade Federal de Santa Maria \\ douglasbouv@gmail.com \\ Rivaldo Mauro de Faria \\ Professor Adjunto do Departamento de Geociências \\ Universidade Federal de Santa Maria \\ rivaldofaria.geo@gmail.com
}

\section{RESUMO}

A Geografia da Saúde é fundamental para o entendimento da evolução dos casos de COVID19, levando a interpretação e a compreensão da dinâmica espacial da ocorrência da doença. É importante compreender que quanto maior for a abrangência espacial do evento, maiores as chances de propagação e maiores são os desafios para vigilância e contenção. Assim, o presente trabalho tem como objetivo compreender a distribuição dos casos confirmados da COVID-19, por bairros e por Regiões Administrativas (RA), na área urbana de Santa Maria, relacionando-a com o Índice de Privação Social (IPS) e identificando possíveis padrões espaciais decorrentes da sua evolução até a décima nona Semana Epidemiológica (SE). A metodologia do trabalho consistiu na sistematização cartográfica e análise de dados referente aos casos confirmados de COVID-19 da $14^{\text {a }}$ SE até a metade da $19^{\mathrm{a}}$, bem como com sua associação com o IPS, elaborado por Spode (2020). Os casos registrados demonstram o avanço da COVID-19 para regiões periféricas de Santa Maria, conduzindo a necessidade de planejamento de estratégias para o combate ao novo Coronavírus.

Palavras-chave: Coronavírus. Índice de Privação Social. Regiões Administrativas. Geografia da Saúde. Geografia Urbana.

\begin{abstract}
Health Geography is fundamental for understanding the evolution of COVID-19 cases, leading to the interpretation and understanding of the spatial dynamics of the occurrence of the disease. It is important to understand that the greater the spatial scope of the event, the greater the chances of spreading and the greater the challenges for surveillance and containment. This study aims to understand the distribution of confirmed cases of COVID-19, by neighborhoods and by Administrative Region (AR), in the urban area of Santa Maria, relating it to the Social Deprivation Index (SDI) and identifying possible spatial patterns resulting from its evolution until the nineteenth Epidemiological Week (EW). The methodology
\end{abstract}

Recebido em: 08/05/2020

Aceito para publicação em: 21/05/2020. 


\begin{abstract}
of the work consisted of cartographic systematization and data analysis referring to confirmed cases of COVID-19 from the 14th EW to the 19th, as well as its association with the SDI, prepared by Spode (2020). The recorded cases demonstrate the advance of COVID-19 to peripheral regions of Santa Maria, leading to the need to plan strategies to combat the new Coronavirus.
\end{abstract}

keywords: Coronavirus. Social Deprivation Index. Administrative Regions. Health Geography. Urban Geography.

\title{
INTRODUÇÃO
}

A Geografia da Saúde se ocupa de diversas questões que a atrelam ao entendimento das diferentes enfermidades que podem ocorrer em determinados territórios, bem como se importa com a abordagem dos condicionantes para as evoluções de quadros epidêmicos/pandêmicos e para o planejamento e gestão de estratégias de promoção de saúde e qualidade de vida. Neste sentido, em tempos do novo Coronavírus, utilizar ferramentas que permitam o entendimento e a espacialização dos casos confirmados, suspeitos e/ou descartados tem impacto decisivo na promoção de estratégias de enfrentamento da doença, tornando a Geografia da Saúde central a essa discussão.

Segundo Junqueira $(2009$, p. 2), "[...] a relação entre a Geografia e a Saúde não é nova, remonta ao final do século XIX, quando era baseada no pensamento Hipocrático [...], e não havia conhecimento dos processos de transmissão de doenças". A partir dessa aproximação, pensar a saúde e sua relação com o território passou a ser primordial no entendimento da evolução de enfermidades, fazendo com que geógrafos e profissionais da saúde busquem trabalhar de forma articulada pela resolução de problemas que atingem a humanidade. "[...] Em 1930, a Geografia se aproxima da Epidemiologia na busca de estabelecer, para diversas doenças, as suas redes de causalidades, e a produção de trabalhos é marcada por forte influência das descobertas bacteriológicas" (JUNQUEIRA, 2009, p. 4). Tal fato aproxima-se na forma de entendimento e de abordagem da atual pandemia, causada por um vírus.

A problematização da distribuição da ocorrência da COVID-19 e a busca por mitigação dos impactos causados pelo vírus, especialmente, em regiões periféricas e com mais elevada vulnerabilidade social em Santa Maria, pode ser pensada a partir da análise espacial e cartográfica das ocorrências, colaborando com o entendimento do processo de espraiamento da contaminação. A produção cartográfica e a interpretação desses resultados podem subsidiar a promoção da saúde e se torna uma ferramenta muito importante às discussões de Geografia da Saúde.

O município de Santa Maria é localizado na região central do estado do Rio Grande do Sul. Caracterizase por ser uma cidade média e por desempenhar papel de centro regional no contexto da rede urbana estadual, exercendo papel importante na região em que se encontra, principalmente oferecendo serviços especializados, como de saúde e educação e também no comércio (ROCHA, 2011).

Em termos de formação socioespacial, Santa Maria se desenvolveu através de eventos sociais e históricos que contribuíram para a construção do território do município. A implantação da ferrovia, ainda no século XIX, pode ser considerado um exemplo de evento histórico e social que é determinante para a formação socioespacial de Santa Maria. Além da ferrovia, alguns objetos implantados ao longo da evolução histórica da cidade e os diferentes usos que cada objeto proporcionou e ainda proporciona ao território, devem ser entendidos em relação a construção histórica da cidade, como é o caso da Universidade Federal de Santa Maria (DEGRANDI, 2012; SPODE, 2020).

No contexto atual, Santa Maria se revela como uma cidade com expressivas desigualdades socioespaciais. São inúmeros os problemas relacionados a pobreza, como é o caso das ocupações irregulares, fenômeno que vem se reproduzindo historicamente na cidade, sobretudo nos bairros periféricos, em áreas de elevada privação social (SPODE, 2020).

Em face disso, pensar a vulnerabilidade social, por meio do Índice de Privação Social (IPS), atrelada à presença do novo Coronavírus em diferentes unidades geográficas da área urbana de Santa Maria, RS, a saber, bairros e Regiões Administrativas (RA), potencializa a compreensão da evolução da doença na cidade e permite ao poder público e órgãos vinculados a saúde pensarem políticas públicas coerentes com as demandas do território em análise. Partindo dessas premissas, o presente trabalho 
tem como objetivo compreender a distribuição dos casos confirmados da COVID-19, por bairros e por RA, na área urbana de Santa Maria, relacionando-a com o IPS e identificando possíveis padrões espaciais decorrentes da sua evolução até a metade da $19^{a}$ semana epidemiológica (SE) ${ }^{2}$.

\section{MATERIAS E MÉTODOS}

Os dados referentes ao número de casos confirmados de COVID-19 em Santa Maria foram obtidos da Secretaria Municipal da Saúde de Santa Maria por meio do projeto "Enfrentamento da epidemia da COVID-19 no estado do Rio Grande do Sul", sendo aprovado pela Comissão Nacional de Ética em Pesquisa (CONEP), sob o CAAE n 30710520.6.0000.5346.

Para a geocodificação dos casos confirmados de COVID-19, isto é, atribuir uma coordenada geográfica (latitude e longitude) a cada endereço, utilizou-se o complemento "MMQGIS", presente no software QGIS 3.12.0, a fim de espacializar, pontualmente, cada um dos casos confirmados de Santa Maria. Posteriormente, utilizou-se a ferramenta "contagem de pontos em polígono", de acordo com o shapefile de bairros e RA, disponibilizados pelo Instituto de Planejamento de Santa Maria (IPLAN, 2020).

Para o cálculo das taxas de incidência da COVID-19 para 100 mil habitantes, foi utilizada a população residente da tabela 1378, disponível no Banco de Tabelas Estatísticas (SIDRA) do Instituto Brasileiro de Geografia e Estatística (IBGE, 2010), com unidade territorial de bairros e subdistritos ${ }^{3}$ para o mapa da taxa de incidência por bairro e RA, respectivamente. O cálculo da taxa de incidência de COVID-19 foi realizado na calculadora de campo do QGIS.

A elaboração do IPS da área urbana de Santa Maria ocorreu em três etapas que estão descritas no trabalho de Spode (2020) e compreendem, de maneira geral, ao processo de seleção das variáveis, retiradas do Censo Demográfico do IBGE de 2010, ao nível dos setores censitários, para elaboração dos indicadores sociais, posteriormente, a padronização e a ponderação desses indicadores. Foram selecionados cinco indicadores sociais para compor o IPS, que avaliam três dimensões da privação social: a dimensão educação, a dimensão renda e a dimensão domicílio-saneamento. Esses indicadores são: taxa de alfabetização; taxa de pessoas sem rendimento nominal mensal e até $1 / 2$ salário mínimo; rendimento médio por domicílio particular permanente; taxa dos domicílios particulares permanentes com esgotamento sanitário via vala; taxa dos domicílios particulares permanentes com cinco ou mais moradores.

A padronização se deu através da técnica do mínimo e máximo, onde os valores de cada setor censitário utilizado foram transformados em uma escala que varia entre 0 e 1 , com os valores mais próximos de 1 correspondendo a uma privação muito alta. Para a ponderação dos indicadores, utilizouse o Método de Análise Hierárquica (Analytic Hierarchy Process), que utiliza um modelo chamado "matriz de comparações par a par", onde cada indicador é comparado entre si. Desse modo, a matriz de comparação permitiu que fossem atribuídos pesos diferentes para cada indicador selecionado, como pode ser identificado no quadro 1.

Quadro 1 - Indicadores selecionados com respectivos pesos.

\begin{tabular}{|l|l|}
\hline Indicadores & Pesos \\
\hline Taxa de alfabetização & 0,45 \\
\hline Taxa de Pessoas sem rendimento nominal mensal e até $1 / 2$ salário & 0,24 \\
\hline Rendimento médio por domicílio particular permanente & 0,16 \\
\hline Taxa dos domicílios particulares permanentes com esgotamento sanitário via vala & 0,09 \\
\hline Taxa dos domicílios particulares permanentes com 5 ou mais moradores & 0,05 \\
\hline
\end{tabular}

Fonte: IBGE (2011).

\footnotetext{
2 Por convenção internacional as semanas epidemiológicas são contadas de domingo a sábado. A primeira semana do ano é aquela que contém o maior número de dias de janeiro e a última a que contém o maior número de dias de dezembro (BRASIL, 2020).

${ }^{3}$ As Regiões Administrativas de Santa Maria, elaboradas pelo Instituto de Planejamento de Santa Maria (IPLAN) e os Subdistritos do Instituto Brasileiro de Geografia e Estatística (IBGE), agrupam os bairros da mesma maneira. Por isso, utilizou-se a unidade territorial dos subdistritos do IBGE para adquirir a população residente por RA. Para o bairro "Sem Denominação", que passou a constituir a área urbana de Santa Maria em 2015, compondo a Região Administrativa Sul, realizou-se uma estimativa visual a partir do número de residência de uma imagem de satélite do ano de 2010, relacionando com o número médio de residentes por domicílio do mesmo ano na área em questão. DOl:http://dx.doi.org/10.14393/Hygeia0054554 Hygeia Edição Especial: Covid-19, Jun./2020 p.441- 449, pág. 443
} 
Finalmente, os dados foram modelados no Microsoft Office Excel e posteriormente inseridos em ambiente SIG. O mapa da taxa de incidência e do IPS foram elaborados no QGIS 3.12.0 e finalizados no compositor de impressão do mesmo software.

\section{RESULTADOS E DISCUSSÃO}

\section{DISTRIBUIÇÃO DA COVID-19 NO DISTRITO SEDE DE SANTA MARIA, RS}

O primeiro caso de COVID-19, em Santa Maria, foi confirmado no dia 21 de março de 2020 (12 ${ }^{\mathrm{a}}$ SE). Posteriormente a isso, uma rede de monitoramento foi organizada para observar a evolução dos casos. No dia 31 de março de 2020, já havia oito casos confirmados, localizados nos seguintes bairros: Camobi (3), Centro (2), Pé-de-Plátano (2) e Lorenzi (1). Em 19 de abril de 2020, houve um crescimento do número de casos, ampliando a incidência de 4 para 14 bairros, a saber, Centro (4), Camobi (3), Duque de Caxias (2), Nossa Senhora de Lourdes (2), Passo D’areia (2), Pé-de-Plátano (2), Boi Morto (1), Caturrita (1), Juscelino Kubitschek (1), Lorenzi (1), Nossa Senhora das Dores (1), Nossa Senhora do Rosário (1), Nossa Senhora Medianeira (1) e Salgado Filho (1). Já no dia 05 de maio de 2020, a dispersão de casos de COVID-19 chegou a 39 confirmados, presentes em 19 dos 42 bairros de Santa Maria. Os bairros Centro (6), Passo D'areia (4), Urlândia (4), Camobi (3), Nossa Senhora de Lourdes (3), Nossa Senhora Medianeira (3), Caturrita (2), Duque de Caxias (2), Pé-de-Plátano (2), Boi Morto (1), Juscelino Kubitschek (1), Lorenzi (1), Nossa Senhora das Dores (1), Nossa Senhora de Fátima (1), Nossa Senhora do Perpétuo Socorro (1), Nossa Senhora do Rosário (1), Presidente João Goulart (1), Salgado Filho (1) e São João (1), são aqueles que apresentavam casos confirmados de COVID-19 na data apontada. A Tabela 1 ilustra as informações apresentadas nas três datas mencionadas, sintetizando a evolução da contaminação por novo Coronavírus nas semanas epidemiológicas $14^{\mathrm{a}}$ (31/03/2020), 17 $7^{a}(19 / 04 / 2020)$ e 19a (05/05/2020).

Tabela 1 - Evolução dos casos de COVID-19 na área urbana de Santa Maria

\begin{tabular}{|c|c|c|c|}
\hline \multirow[b]{2}{*}{ Bairros } & \multicolumn{3}{|c|}{ Casos confirmados de COVID-19 } \\
\hline & $\begin{array}{c}31 / 03 / 2020 \\
\left(14^{\mathrm{a}} \mathrm{SE}\right)\end{array}$ & $\begin{array}{c}19 / 04 / 2020 \\
\left(17^{\mathrm{a}} \mathrm{SE}\right)\end{array}$ & $\begin{array}{c}05 / 05 / 2020 \\
\left(19^{\mathrm{a}} \mathrm{SE}\right)\end{array}$ \\
\hline Boi Morto & 0 & 1 & 1 \\
\hline Camobi & 3 & 3 & 3 \\
\hline Caturrita & 0 & 1 & 2 \\
\hline Centro & 2 & 4 & 6 \\
\hline Duque de Caxias & 0 & 2 & 2 \\
\hline Juscelino Kubitschek & 0 & 1 & 1 \\
\hline Lorenzi & 1 & 1 & 1 \\
\hline N. Sra. das Dores & 0 & 1 & 1 \\
\hline N. Sra. De Fátima & 0 & 0 & 1 \\
\hline N. Sra. de Lourdes & 0 & 2 & 3 \\
\hline N. Sra. do Perpétuo Socorro & 0 & 0 & 1 \\
\hline N. Sra. do Rosário & 0 & 1 & 1 \\
\hline N. Sra. Medianeira & 0 & 1 & 3 \\
\hline Passo D'areia & 0 & 2 & 4 \\
\hline Pé-de-Plátano & 2 & 2 & 2 \\
\hline Presidente João Goulart & 0 & 0 & 1 \\
\hline Salgado Filho & 0 & 1 & 1 \\
\hline São João & 0 & 0 & 1 \\
\hline Urlândia & 0 & 0 & 4 \\
\hline TOTAL & 8 & 23 & 39 \\
\hline
\end{tabular}

Org.: Maurício Rizzatti (2020). 
Evolução e periferização da covid-19 na área urbana de Santa Maria, RS: traçando padrões espaciais
Maurício Rizzatti Pedro Leonardo Cezar Spode Natália Lampert Batista Douglas Bouvier Erthal Rivaldo Mauro de Faria

Para um maior detalhamento e espacialização do atual contexto da COVID-19 na área urbana de Santa Maria, apresenta-se a Figura 1. Nela está detalhada a distribuição dos casos confirmados, bem como a taxa de incidência por bairro (para 100 mil habitantes). Tais dados evidenciam uma periferização do Coronavírus, levando a necessidade de planejar a gestão das estratégias de saúde para a mitigação deste processo.

Desde a $12^{\text {a }} \mathrm{SE}$, quando ocorreu o registro do primeiro caso confirmado de COVID-19 em Santa Maria, foi possível observar que os bairros Centro e Camobi apresentaram-se como foco inicial do vírus, com uma maior incidência da doença, fato atribuído a maior circulação de pessoas e disponibilidade de serviços, conforme exposto na Tabela 1. Porém, o número de casos nestes bairros encontra-se, de acordo com os dados do dia 05 de maio de 2020 (19a SE), em processo de estabilização. Ainda ocorre um crescimento no Centro, porém menos acentuado do que observado nos demais bairros; já o bairro Camobi se mantem estagnado em número de notificações.

$\mathrm{Na} 17^{\mathrm{a}} \mathrm{SE}$, evidencia-se o início do processo de periferização do vírus, com surgimento do primeiro caso confirmado nos bairros Boi Morto, Caturrita, Juscelino Kubitschek e Salgado Filho. Também ocorre um aumento dos casos nas áreas circunvizinhas ao Centro, como nos bairros Nossa Senhora das Dores, Nossa Senhora de Lourdes, Nossa Senhora Medianeira e Nossa Senhora do Rosário. Dessa forma, há uma mudança na tendência espacial da distribuição da COVID-19. Essa constatação ganha força na $19^{\mathrm{a}} \mathrm{SE}$, em que o crescimento dos casos em números absolutos se torna maior nos bairros considerados periféricos e, posteriormente, destacados no próximo item do trabalho.

Figura 1 - Taxa de incidência (por 100 mil habitantes) e número de casos confirmados de COVID-19 por bairro na área urbana de Santa Maria, RS, em 05 de maio de 2020

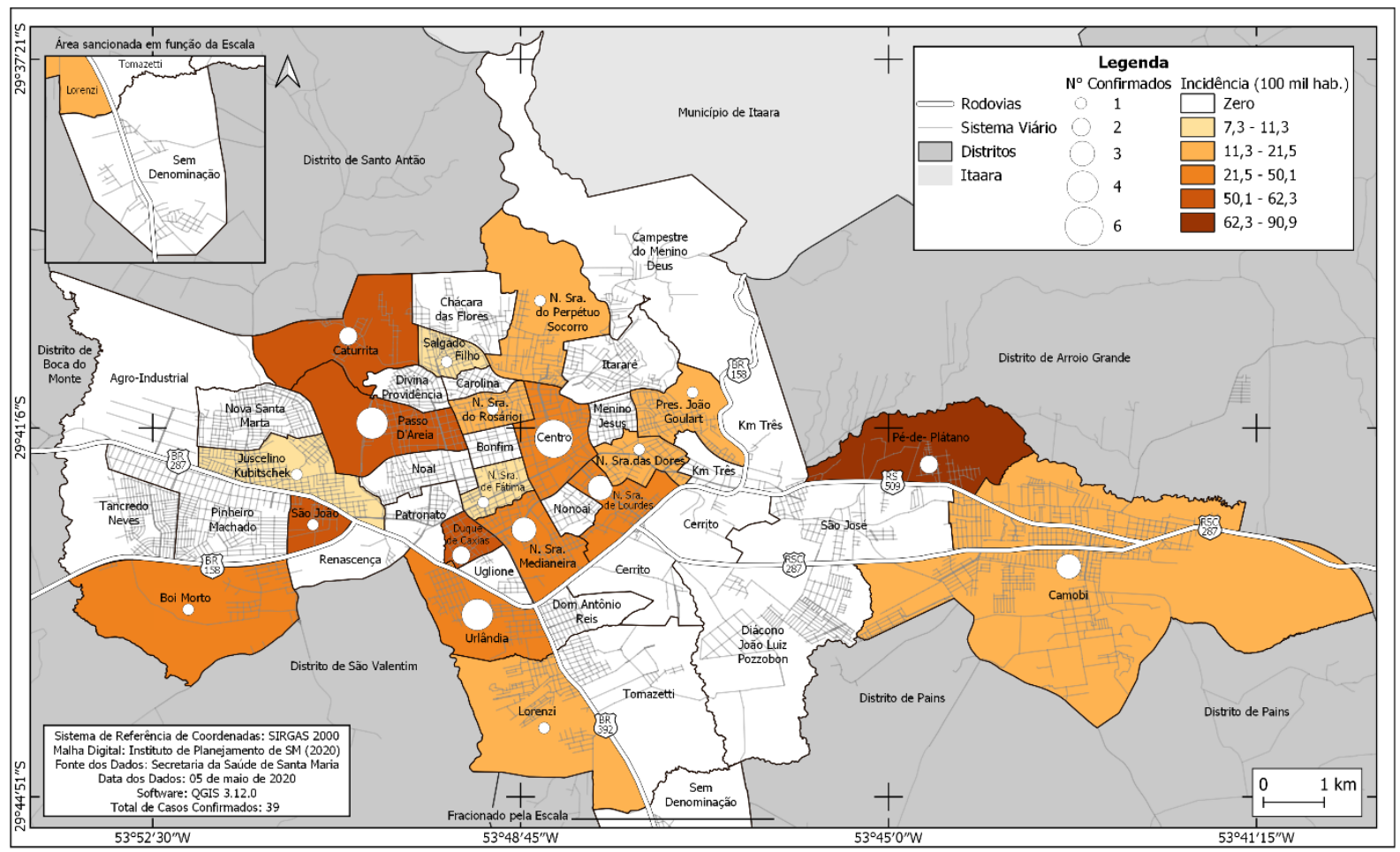

Elaboração: Maurício Rizzatti (2020).

A Vigilância Epidemiológica de Santa Maria está se organizando para adotar as RA como Regiões Sanitárias para o enfrentamento da COVID-19 na cidade de Santa Maria. A Figura 2 e a Tabela 2 ilustram como os bairros destacados anteriormente se relacionam com estas unidades espaciais, bem como destaca a incidência por 100 mil habitantes dos casos da doença em cada RA. Além disso, demonstra o número de unidades de saúde que serão responsáveis pela gestão da pandemia em nível local. 
Evolução e periferização da covid-19 na área urbana de Santa Maria, RS: traçando padrões espaciais
Maurício Rizzatti Pedro Leonardo Cezar Spode Natália Lampert Batista Douglas Bouvier Erthal Rivaldo Mauro de Faria

Salienta-se que as RA Centro-Oeste e Oeste são os pontos extremos na taxa de incidência do novo Coronavírus, apresentando a maior e a menor incidência por 100 mil habitantes, respectivamente. Em contraste a isso, o menor número de unidades de saúde estão localizadas na RA Centro-Oeste e o maior na RA Oeste, levando a necessidade da compreensão da distribuição espacial desses dois fatores para a gestão da políticas de contenção do vírus, ou seja, pensar a distribuição de recursos humanos nessas unidades de saúde, entre outras medidas a serem adotadas.

A RA Centro-Oeste, que totaliza seis casos confirmados, possui uma incidência de 26,91 por 100 mil habitantes. Na RA Centro Urbano, que conta com catorze casos confirmados de COVID-19, a taxa de incidência, que é de 23,41 para 100 mil habitantes, é menor que RA Centro-Oeste, devido ao maior contingente populacional residente. Com valor semelhante ao Centro Urbano, a RA Sul, com cinco casos confirmados e incidência de 23,07 , merece destaque, pois sua significativa ampliação deve-se ao acentuado surgimento de casos no bairro Urlândia; inexistindo contaminados pelo novo Coronavírus na $17^{\mathrm{a}} \mathrm{SE}$, passando para quatro casos na $19^{\mathrm{a}} \mathrm{SE}$.

Já a RA Centro-Leste, com dois casos confirmados, conta com uma taxa de 16,43 para 100 mil habitantes, aproximando-se das RA Norte e Leste com quatro e três casos confirmados, respectivamente, correspondendo as taxas de 14,39 e 13,75 por 100 mil habitantes. Deve-se enfatizar, todavia, que nas RA Leste e Centro-Leste, não há ampliação dos casos desde a $14^{\mathrm{a}} \mathrm{SE}$, em contraponto com as demais que apresentam crescimento.

Figura 2 - Taxa de incidência (por 100 mil habitantes) e número de casos confirmados de COVID-19 por RA na área urbana de Santa Maria, RS, em 05 de maio de 2020

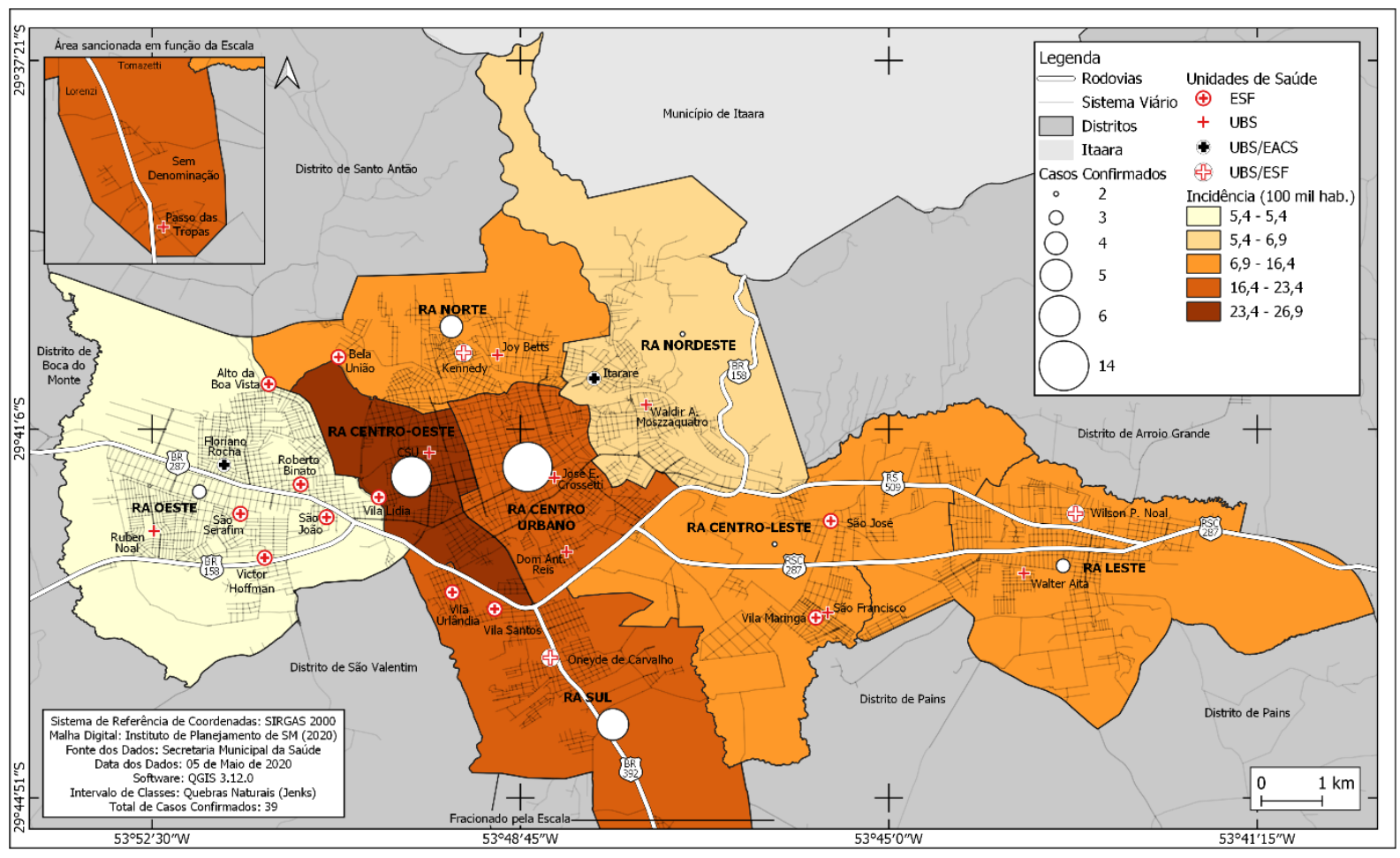

Elaboração: Maurício Rizzatti (2020).

Com os menores índices de incidência de COVID-19, sobressaem-se as RA Nordeste e Oeste. Apresentando três casos e 6,94 por 100 mil habitantes e dois casos e 5,44 por 100 mil habitantes de números absolutos e de taxa, respectivamente. 
Tabela 2 - Taxa de incidência de COVID-19 (por 100 mil hab.), bairros que compõem as RA e número de unidades de saúde por RA, em 05 de maio de 2020

\begin{tabular}{|c|c|c|c|}
\hline $\begin{array}{l}\text { Região } \\
\text { Administrativa } \\
\text { (RA) }\end{array}$ & $\begin{array}{c}\text { Taxa de } \\
\text { Incidência } \\
\text { por RA (por } \\
100 \text { mil hab.) } \\
\text { em } \\
05 / 05 / 2020\end{array}$ & Bairros que compõem a RA & $\begin{array}{l}N^{\circ} \text { de unidades } \\
\text { de saúde por RA } \\
\text { (UBS, ESF e } \\
\text { EACS) }\end{array}$ \\
\hline Centro Urbano & 23,41 & $\begin{array}{l}\text { Bonfim, Centro, N. Sra. de Fátima, N. Sra. } \\
\text { de Lourdes, N. Sra. do Rosário, N. Sra. } \\
\text { Medianeira e Nonoai }\end{array}$ & 2 \\
\hline Centro-Leste & 16,43 & $\begin{array}{l}\text { Cerrito, Diácono J. L. Pozzobon, Pé-de- } \\
\text { Plátano e São José }\end{array}$ & 3 \\
\hline Centro-Oeste & 26,91 & $\begin{array}{l}\text { Duque de Caxias, Noal, Patronato, Passo } \\
\text { D'areia e Uglione }\end{array}$ & 2 \\
\hline Leste & 13,75 & Camobi & 2 \\
\hline Nordeste & 6,94 & $\begin{array}{l}\text { Campestre do Menino Deus, Itararé, Km } 3 \text {, } \\
\text { Menino Jesus, N. Sra. das Dores e } \\
\text { Presidente João Goulart }\end{array}$ & 2 \\
\hline Norte & 14,39 & $\begin{array}{l}\text { Carolina, Caturrita, Chácara das Flores, } \\
\text { Divina Providência, N. Sra. do Perpétuo } \\
\text { Socorro e Salgado Filho }\end{array}$ & 3 \\
\hline Oeste & 5,44 & $\begin{array}{l}\text { Agro-Industrial, Boi Morto, Juscelino } \\
\text { Kubistchek, Nova Santa Marta, Pinheiro } \\
\text { Machado, Renascença, São João e } \\
\text { Tancredo Neves }\end{array}$ & 7 \\
\hline Sul & 23,07 & $\begin{array}{l}\text { Dom Antônio Reis, Lorenzi, Sem } \\
\text { Denominação, Tomazetti e Urlândia }\end{array}$ & 3 \\
\hline
\end{tabular}

Org.: Maurício Rizzatti e Natália Lampert Batista (2020).

Os casos registrados demonstram a dispersão da COVID-19 para regiões periféricas de Santa Maria, conduzindo a necessidade de planejamento de estratégias para o combate ao novo Coronavírus. Para aprofundar tal abordagem, na sequência, abordou-se a periferização da COVID-19, com base na sua correlação com o IPS, que identifica as áreas de maior pobreza na cidade e privadas de recursos. É importante compreender que quanto maior for a abrangência espacial do evento, maiores as chances de propagação e, consequentemente, os desafios para vigilância e contenção.

\section{PERIFERIZAÇÃO DA COVID-19 NO DISTRITO SEDE DE SANTA MARIA, RS}

A difusão espacial do vírus, como diversos estudos vem demonstrando, desloca-se das áreas mais densas, em termos de fluxos, para as áreas menos densas, com menor fluxo de pessoas e objetos. Esse padrão pode ser observado no estado de São Paulo ou no Rio Grande do Sul, por exemplo, além de países como os Estados Unidos e Itália. Como não poderia ser diferente, esse comportamento parece, também, ocorrer nas cidades médias, como Santa Maria, com os casos se concentrando, primeiramente, nos bairros de maior fluxo, para no segundo momento, avançar para as áreas de menor fluxos, nas periferias da cidade.

Esse padrão espacial pode ser identificado através dos dados expostos, que demonstram que os primeiros bairros com casos confirmados foram Centro e Camobi, locais de maior circulação de pessoas, como também, com maior densidade de comércios e serviços na cidade. Nos bairros citados, como pode ser conferido no mapa do IPS da Figura 3, a privação social é muito baixa, salvo as porções norte e a COHAB Fernando Ferrari em Camobi, duas áreas que apresentam expressivas situações de privação. O bairro Camobi, cabe destacar, abriga a Universidade Federal de Santa Maria, além da ALA 4 (antiga Base Aérea de Santa Maria) e do Aeroporto Civil, o que o torna alvo de intenso fluxo populacional. 
Evolução e periferização da covid-19 na área urbana de Santa Maria, RS: traçando padrões espaciais
Maurício Rizzatti Pedro Leonardo Cezar Spode Natália Lampert Batista Douglas Bouvier Erthal Rivaldo Mauro de Faria

Figura 3 - Índice de Privação Social por setor censitário e número de casos confirmados de COVID19 por bairro em Santa Maria, RS, em 05 de maio de 2020

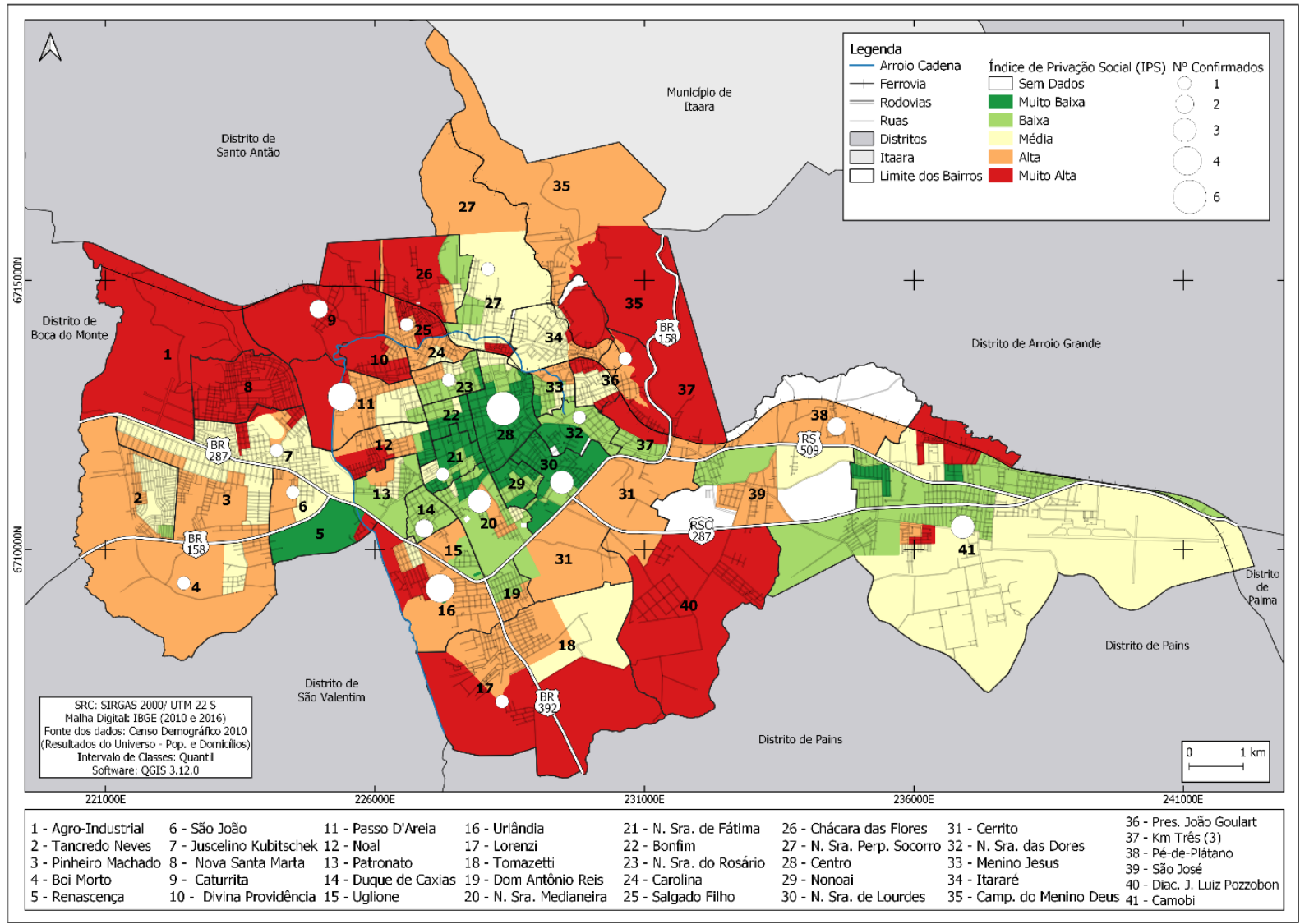

Fonte: SPODE, 2020 - adaptado.

Elaboração: Maurício Rizzatti, 2020

Por outro lado, embora o Centro permaneça registrando casos confirmados, verifica-se que o vírus vem ganhando terreno pelas áreas periféricas, em bairros como Urlândia e Lorenzi, na RA Sul, também no Salgado Filho e Caturrita, na RA Norte, além de bairros como Passo D'areia (RA Centro-Oeste), São João e Boi Morto (RA Oeste) e Presidente João Goulart (RA Nordeste). São essas áreas, Sul, Norte e Oeste, as mais carenciadas da cidade de Santa Maria, como pode ser verificado no mapa (Figura 2), embora não em sua totalidade.

Desse modo, o que o IPS nos sugere, é que nas enormes manchas de pobreza, representadas nas áreas em vermelho no mapa, as condições para enfrentamento da doença são mais limitadas, em vista de todas as privações dessas áreas. Nessas áreas, cujo adensamento ocupacional das residências é extremamente elevado, as medidas de isolamento social e de quarentena se tornam tarefas impraticáveis. No bairro João Goulart (RA Nordeste), por exemplo, um dos setores censitários apresenta taxa de 16,92 moradores por domicílio, assim como no bairro Salgado Filho (RA Norte), com 15,72, conforme os dados demonstrados por Spode (2020).

Ademais, são essas áreas periféricas as que possuem os maiores problemas relacionados a infraestrutura urbana. Essa realidade é evidenciada, especialmente, nas áreas de ocupação irregular na cidade, privadas dos recursos mais basilares, como o saneamento básico e a própria moradia. Essas áreas irregulares se localizam, principalmente, nas margens do Arroio Cadena e dos trilhos da ferrovia, em sua maioria abandonados, em bairros como Salgado Filho, Carolina, Caturrita, Passo D'Areia, Urlândia, Lorenzi, entre outros bairros de alta privação social.

Nesse sentido, se no primeiro momento a COVID-19 se manifesta nas áreas de baixa privação, em núcleos densificados, com maiores fluxos, no momento posterior, a doença atinge as áreas mais pobres, habitadas por homens mais lentos, como diria Milton Santos (2006), cuja estrutura urbana é 
historicamente precarizada. Na cidade de Santa Maria, como coloca Spode (2020), os pobres são distribuídos geograficamente em todas as áreas, no entanto, concentrando-se em sua maioria nas regiões periféricas, especialmente nas áreas de influência ferroviária ou do Arroio Cadena, áreas cuja incidência do novo Coronavírus tende a se intensificar.

\section{CONSIDERAÇÕES FINAIS}

O presente trabalho traz como contribuição uma discussão, ainda em andamento devido a vigência pandêmica, sobre a evolução espacial da COVID-19 na área urbana de Santa Maria, promovendo uma argumentação articulada com os debates da Geografia da Saúde e buscando reduzir os impactos do novo Coronavírus em nível local. Os dados observados ao longo do texto, permitem evidenciar que os bairros Centro e Camobi se encontram em processo de estabilização da infecção de seus moradores, apesar dos maiores fluxos de pessoas e densidades de serviços. Em contrapartida, os bairros periféricos estão em ascensão nas taxas de incidência de casos confirmados da enfermidade. Essas averiguações são percebidas ao comparar as SE citadas no decorrer do trabalho e as produções cartográficas apresentadas.

Ressalta-se, também, que a adoção das RA, como Regiões Sanitárias, para o planejamento das estratégias em saúde em Santa Maria, pode se mostrar eficiente, desde que articuladas as demandas dos contaminados com as unidades de serviços de saúde e disponibilidade de recursos humanos para atendimento dessa população. Um fato preocupante no contexto santa-mariense é a sobreposição do número de casos confirmados com uma privação social elevada, pois compromete a promoção da saúde na medida em que as populações não conseguem cumprir as medidas necessárias de prevenção: isolamento social e quarentena. Tal fato conduz a necessidade da adoção de indicadores que colaborem com o enfrentamento da pandemia de forma que se preserve a vida dos moradores de Santa Maria, reduzindo os impactos, em especial, nas populações carentes, que tendem a sofrer mais com o novo Coronavírus.

\section{AGRADECIMENTOS}

O trabalho foi realizado no âmbito do projeto "Observatório de Dados da COVID-19", da Universidade Federal de Santa Maria, como colaboração à vigilância e através do qual se teve acesso aos dados.

O presente trabalho foi realizado com apoio da Coordenação de Aperfeiçoamento de Pessoal de Nível Superior - Brasil (CAPES) - Código de Financiamento 001 e ao Programa Nacional de Pós-Doutorado (PNPD - CAPES).

\section{REFERÊNCIAS}

BRASIL. Calendário de notificação para o ano de 2020. Brasília, DF: Ministério da Saúde, 2020.

DEGRANDI, J. O. Verticalidades e horizontalidades nos usos do território de Santa Maria-RS. Tese (Doutorado), Santa Cruz do Sul, RS. Universidade de Santa Cruz do Sul, 2012.

IBGE. Base de informações do Censo Demográfico 2010: resultados do universo por setor censitário. Rio de Janeiro: Centro de Documentação e Disseminação de Informações, 2011.

JUNQUEIRA, R. D. Geografia Médica ou da Saúde. Hygeia: Revista Brasileira de Geografia Médica e da Saúde (Uberlândia), v. 5, p. 1-10, 2009. Disponível em: http://www.seer.ufu.br/index.php/hygeia. Acesso em: 05 de mai. 2020.

ROCHA, L. H. M. da, Padrão locacional da estrutura social: segregação residencial em Santa Maria - RS. Tese (Doutorado), Florianópolis, SC. Universidade Federal de Santa Catarina, 2011.

SANTOS, M. A natureza do espaço: técnica e tempo, razão e emoção. 2. reimpr. São Paulo: Editora da Universidade de São Paulo, v. 1, 2006.

SPODE, P. L. C. Pobreza e privação social na área urbana de Santa Maria, Rio Grande do Sul: uma análise a partir dos usos do território. Dissertação (mestrado) - Universidade Federal de Santa Maria, Centro de Ciências Naturais e Exatas, Programa de Pós-Graduação em Geografia e Geociências, RS, 2020. 